\title{
Modeling of static deformations and dynamics of localized rotation in a chain of finite size particles
}

\author{
A. A. Vasiliev ${ }^{1, \dagger}$, A.E. Miroshnichenko ${ }^{2}$ \\ †aleksey.vasiliev@gmail.com \\ ${ }^{1}$ Tver State University, Sadoviy per., 35, Tver, 170002, Russia \\ ${ }^{2}$ School of Engineering and Information Technology, University of New South Wales Canberra, ACT 2600, Australia

\begin{abstract}
In beam-like structures and granular media the rotations are usually quite small. Linear generalized continuum models were developed and successfully applied for the modelling of such deformations. But, they do not describe full continuous rotations. The development of generalized continuum models describing not only small oscillations, but full continuous rotations as well is quite challenging and an open problem. This manuscript extends the results by Vasiliev A. A, Dmitriev S. V. Discrete and Multifield Models of a Cosserat Chain: One-Period and Two-Period Solutions. Russian Physics Journal. 59, 961 (2016). We derive and analyse three types of discrete models: i) nonlinear springs model, ii) linearized model with respect to displacement of particles and full sine-dependence of the angle of rotations; and iii) linearized one with respect to both, displacements and rotations. The latter is the discrete analogue of the classical Cosserat model. As a test example, we consider a chain of finite size particles with fixed boundaries in viscous medium with an applied momentum load to the central particle. By employing continuous Cosserat model, we find an approximated stationary solution for the full and reduced discrete Cosserat models. Numerically calculated static deformations of the chain under small momentum loads by using all three models are very similar. Under strong load localized rotation is observed. It is not described by the linear model (iii). Our results indicate the possibility of modelling of localized rotations in the nonlinear model (i) and the model containing nonlinear sine-dependence of the angle of rotation (ii).
\end{abstract}

Keywords: Cosserat chain, model, rotation.

\section{Introduction}

The modelling of continuous media and discrete lattices with rotational degrees of freedom is one of important problems of generalized continuum mechanics. In beam-like structures and granular media the rotations are usually quite small $[1,2]$. For modelling of such deformations there were developed linear models, which takes into account small oscillations only $[3,4]$. The development of generalized mechanics models describing not only small oscillations, but full continuous rotations as well is quite challenging and an open problem. In the current paper we extend the results presented in [5].

Currently, there is huge interest in development of mechanical metamaterials, i.e. materials with special and unique properties, which are not present in natural materials. From this perspective, among general Cosserat solids there is a special type of models where the second derivative of rotational degree of freedom in corresponding equation for rotations is absent, so-called reduced Cosserat models [6, 7]. Understanding of mathematical and physical properties of such models, as well as general approach to describe the behavior of such media is quite nontrivial and interesting task.

In the development of generalized mechanics, as well as classical mechanics of deformable bodies, there are two common approaches: phenomenological and structural ones.
Phenomenological approach is quite generic, but it is based on actual experimental data. Structural approach provides in-depth understanding of a developing theory, possibilities for its practical realization, experimental verifications and numerical modelling $[8,9]$.

\section{Models}

We consider 1D discrete lattice of particles of finite sizes, where their rotational degrees of freedom are taken into account.

The kinetic energy can be expressed in the following form

$$
K=1 / 2 m(\dot{u}+\dot{v})+1 / 2 j \dot{\varphi},
$$

where $m$ is a mass, $j$ is a moment of inertia, $u$ and $v$ are longitudinal and transverse displacements, and $\varphi$ describes rotational degree of freedom in the form of either small oscillations or full continuous rotations of particles.

In the basic nonlinear model let's consider elastic interaction with the potential energy

$$
P=1 / 2 E\left(L-L_{0}\right)^{2},
$$

where $E$ is stiffness of an element, $L_{0}$ is initial length of the element and $L$ is its length after the deformation.

The initial length $L_{0}$ is found according to the following expressions 


$$
\begin{gathered}
X_{0}=x_{0}+r_{0} \cos \alpha_{0}, \quad Y_{0}=y_{0}+r_{0} \sin \alpha_{0} \\
X_{1}=x_{1}+r_{1} \cos \alpha_{1}, \quad Y_{1}=y_{1}+r_{1} \sin \alpha_{1} ; \\
L_{0}=\sqrt{\left(X_{1}-X_{0}\right)^{2}+\left(Y_{1}-Y_{0}\right)^{2}}
\end{gathered}
$$

where $\left[X_{0}, Y_{0}\right],\left[X_{1}, Y_{1}\right]$ are coordinates of connected nodes, $\left[x_{0}, y_{0}\right],\left[x_{1}, y_{1}\right]$ are coordinates of the centers of linked particles, $r_{0}, r_{1}$ are distances from centers to edges, $\alpha_{0}, \alpha_{1}$ are the angles between radius-vectors pointing from the center of particles towards the edges and $x$-axis along the particles centers.

By defining the displacements as $\left[u_{0}, v_{0}\right],\left[u_{1}, v_{1}\right]$, and rotational angles of particles as $\varphi_{0}, \varphi_{1}$ the length of the deformed connecting element is defined as

where

$$
L=\sqrt{\left(X_{1}^{\prime}-X_{0}^{\prime}\right)^{2}+\left(Y_{1}^{\prime}-Y_{0}^{\prime}\right)^{2}} .
$$

$$
\begin{array}{cc}
X_{0}^{\prime}=x_{0}+u_{0}+r_{0} \cos \left(\alpha_{0}+\varphi_{0}\right), & Y_{0}^{\prime}=y_{0}+v_{0}+r_{0} \sin \left(\alpha_{0}+\varphi_{0}\right), \\
X_{1}^{\prime}=x_{1}+u_{1}+r_{1} \cos \left(\alpha_{1}+\varphi_{1}\right), & Y_{1}^{\prime}=y_{1}+v_{1}+r_{1} \sin \left(\alpha_{1}+\varphi_{1}\right)
\end{array}
$$

are coordinates of the connected edges after deformation.

We take into account momentum loads $M$ in the equations for rotations and viscosity of the medium. Such loads can be easily realized by using external side loads. In realistic systems viscosity is usually present. The corresponding expressions are taken into account in equations $(2,3)$ as well.

We don't present the equations of the nonlinear model because they are quite cumbersome. Below we'll refer to them in accordance with the expression of the potential energy (1).

By linearizing these equations with respect to displacements $u_{k}, v_{k}$ and keeping sine-dependence for rotations $\left(\sin \varphi_{k}\right)$, we derive the following system of equations $m \ddot{u}_{k}=2 h^{2}\left(E_{1}+\frac{(h-2 a)^{2}}{d^{2}} E_{2}\right) \frac{u_{k+1}-2 u_{k}+u_{k-1}}{h^{2}}-\gamma_{u} \dot{u}_{k}$, $m \ddot{v}_{k}=8 \frac{b^{2}}{d^{2}} h^{2} E_{2}\left[\frac{v_{k+1}-2 v_{k}+v_{k-1}}{h^{2}}-\frac{\sin \varphi_{k+1}-\sin \varphi_{k-1}}{2 h}\right]-\gamma_{v} \dot{v}_{k}$, $j \ddot{\varphi}_{k}=2 b^{2}\left[h^{2}\left(E_{1}-\frac{h^{2}}{d^{2}} E_{2}\right) \frac{\sin \varphi_{k+1}-2 \sin \varphi_{k}+\sin \varphi_{k-1}}{h^{2}}+\right.$ $\left.+4 \frac{h^{2}}{d^{2}} E_{2}\left(\frac{v_{k+1}-v_{k-1}}{2 h}-\sin \varphi_{k}\right)\right]+M_{k}-\gamma_{\varphi} \dot{\varphi}_{k}$,

where $2 a, 2 b$ are sizes of the particles along the horizontal and vertical directions, $h$ is the distance between their centers, $d$ is the length of diagonal links, $E_{1}, E_{2}$ are stiffnesses coefficients of horizontal and tilted links, respectively (see Fig.1).

The complete linearization with respect to displacements $u_{k}, v_{k}$ and angles of rotation $\varphi_{k}$ leads to the following set of equations

$$
\begin{aligned}
& m \ddot{u}_{k}=2 h^{2}\left(E_{1}+\frac{(h-2 a)^{2}}{d^{2}} E_{2}\right) \frac{u_{k+1}-2 u_{k}+u_{k-1}}{h^{2}}-\gamma_{u} \dot{u}_{k}, \\
& m \ddot{v}_{k}=8 \frac{b^{2}}{d^{2}} h^{2} E_{2}\left[\frac{v_{k+1}-2 v_{k}+v_{k-1}}{h^{2}}-\frac{\varphi_{k+1}-\varphi_{k-1}}{2 h}\right]-\gamma_{v} \dot{v}_{k}, \\
& j \ddot{\varphi}_{k}=2 b^{2}\left[h^{2}\left(E_{1}-\frac{h^{2}}{d^{2}} E_{2}\right) \frac{\varphi_{k+1}-2 \varphi_{k}+\varphi_{k-1}}{h^{2}}+\right. \\
& \left.+4 \frac{h^{2}}{d^{2}} E_{2}\left(\frac{v_{k+1}-v_{k-1}}{2 h}-\varphi_{k}\right)\right]+M_{k}-\gamma_{\varphi} \dot{\varphi}_{k} .
\end{aligned}
$$

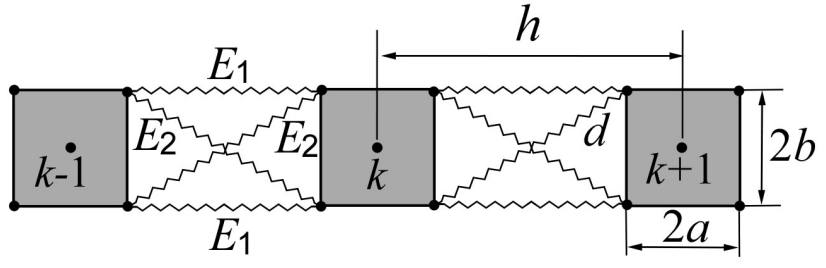

Fig. 1. Schematics of the elementary cell together with the definition of parameters of the system.

These equations are just linearization of the system (2). Formally, they present a discretize version of classical equations for Cosserat solids. The corresponding potential energy can be derived via linearization of the expression $\Delta L=L-L_{0}$ in (1) with respect to displacements $u, v$ and angle of rotation $\varphi$ by assuming that they are small enough. The expression of the potential energy of the links between particles with indices 0 and 1 can be written as

$$
\begin{aligned}
P= & 1 / 2 E\left[\left(u_{1}-u_{0}\right) \cos \gamma+\left(v_{1}-v_{0}\right) \sin \gamma+\right. \\
& +r_{0}\left(\cos \gamma \sin \alpha_{0}-\sin \gamma \cos \alpha_{0}\right) \varphi_{0}- \\
& \left.\left.-r_{1}\left(\cos \gamma \sin \alpha_{1}-\sin \gamma \cos \alpha_{1}\right) \varphi_{1}\right)\right]^{2},
\end{aligned}
$$

where we used the notations $\cos \gamma=\left(X_{1}-X_{0}\right) / L_{0}$, $\sin \gamma=\left(Y_{1}-Y_{0}\right) / L_{0}$, and $\gamma$ describing an initial tilted angle of the link.

In this paper we'll be referring to a model described by the potential energy (1), which is fully nonlinear, as the nonlinear model; a model described by equations (2) as sine-Cosserat model; and a model described by the set of equations (3) as standard linear Cosserat model.

\section{Continuous approximations of steady state solutions in linear full and reduced discrete models}

\subsection{Standard full Cosserat model}

We consider a system with fixed boundaries consisting of odd number of particles $2 N+1$ in viscous medium with an applied angular momentum $M$ at the center. Let's perform linear analysis based on long-wavelength continuous approximation of model (3).

Due to linearity of the system (3) the longitudinal displacements are absent. As a result, we can consider coupled equations for transverse displacements and rotations only. The equations for the steady state without applied moments are the following

$$
\begin{gathered}
\frac{v_{k+1}-2 v_{k}+v_{k-1}}{h^{2}}-\frac{\varphi_{k+1}-\varphi_{k-1}}{2 h}=0, \\
h^{2} D_{1} \frac{\varphi_{k+1}-2 \varphi_{k}+\varphi_{k-1}}{h^{2}}+D_{2}\left(\frac{v_{k+1}-v_{k-1}}{2 h}-\varphi_{k}\right)=0,
\end{gathered}
$$

where we introduced the notations $D_{1}=E_{1}-2\left(h^{2} / d^{2}\right) E_{2}$, $D_{2}=4\left(h^{2} / d^{2}\right) E_{2}$.

To find approximate solutions one can employ the continuum long-wavelength single-field model. From discrete equations (4) and (5) we derive 


$$
v_{x x}-\varphi_{x}=0, \quad h^{2} D_{1} \varphi_{x x}+D_{2}\left(v_{x}-\varphi\right)=0 .
$$

The solution of this system of ordinary differential equations can be easily found and has the form

$$
\begin{gathered}
v(x)=C_{1} x^{3} / 6+C_{2} x^{2} / 2+C_{3} x+C_{4}, \\
\varphi(x)=C_{1} h^{2} D_{1} / D_{2}+C_{1} x^{2} / 2+C_{2} x+C_{3} .
\end{gathered}
$$

The integration constants can be found from the boundary conditions.

Let's put the origin $x=0$ at the central particle, described by index $k=0$. Due to symmetry and fixed boundary condition at $x=L$ we have

$$
v(0)=0, \quad v(L)=0, \quad \varphi(L)=0,
$$

where $L=N h$ is half length of the chain.

The angle of rotation at $x=0$ is undefined. Due to the symmetry we have $v_{1}=-v_{-1}, \varphi_{1}=\varphi_{-1}$. Under such conditions the equation (4) of the discrete model is automatically satisfied and the equation (5) takes the form

$$
2 b^{2}\left[2 h^{2} D_{1} \frac{\varphi_{1}-\varphi_{0}}{h^{2}}+D_{2}\left(\frac{v_{1}}{h}-\varphi_{0}\right)\right]+M=0 .
$$

To find the integration constants one can use the continuous approximation of eq. (9)

$$
2 b^{2}\left[2 h^{2} D_{1} \frac{\varphi(h)-\varphi(0)}{h^{2}}+D_{2}\left(\frac{v(h)}{h}-\varphi(0)\right)\right]+M=0 .
$$

Such condition in continuous theory is called nonlocal one. The solution (7), which satisfies the boundary conditions (8), (10), has the following form

$$
\begin{aligned}
& v(x)=\frac{1}{2 b^{2}} \frac{M x(L-x)\left(6 h^{2} D_{1}+L(L-x) D_{2}\right)}{h\left(24 h^{2} D_{1}^{2}+\left(8 L^{2}+6 h^{2}\right) D_{1} D_{2}+L(2 L-h) D_{2}^{2}\right)}, \\
& \varphi(x)=\frac{1}{2 b^{2}} \frac{M(L-x)\left(12 h^{2} D_{1}+L(L-3 x) D_{2}\right)}{h\left(24 h^{2} D_{1}^{2}+\left(8 L^{2}+6 h^{2}\right) D_{1} D_{2}+L(2 L-h) D_{2}^{2}\right)} .
\end{aligned}
$$

The angle of rotation of the central particle under applied momentum can now be found

$$
\varphi(0)=\frac{1}{2 b^{2}} \frac{M L\left(12 h^{2} D_{1}+L^{2} D_{2}\right)}{h\left(24 h^{2} D_{1}^{2}+\left(8 L^{2}+6 h^{2}\right) D_{1} D_{2}+L(2 L-h) D_{2}^{2}\right)} .
$$

The discrete solution for $v_{k}, \varphi_{k}$ can be obtained from the continuous one with the help of substitution $x=k h$, $k=0,1, \ldots, N$ into continuous solution (11), see above, namely $v_{k}=v(k h), \varphi_{k}=\varphi(k h)$. By substituting it into discrete equations and boundary conditions results in a residue, which allows to estimate the error of the continuous approximation as well as dependence of this error on other parameters. The residue of the discrete equation for displacements (4) is equal to zero, i.e. it is automatically satisfied. At the same time, the residue for the equation (5) is equal to

$$
\Delta_{\varphi}=\frac{1}{2 b^{2}} \frac{M D_{2}^{2} \varepsilon}{24 \varepsilon^{2} D_{1}^{2}+\left(8+6 \varepsilon^{2}\right) D_{1} D_{2}+(2-\varepsilon) D_{2}^{2}},
$$

where the notation $\varepsilon=h / L$ is introduced, or by taking into account that $L=N h, \varepsilon=1 / N$.

The boundary conditions $v_{0}=0, v_{N}=0, \varphi_{N}=0$ of the discrete model (9) are automatically satisfied.
Note, that for $\varepsilon \rightarrow 0$, i.e. when the length of a cell tends to zero with respect to the total length $h / L \rightarrow 0$, the residue (12) vanishes. It can be explained due to the fact that for $h \rightarrow 0$ the difference derivatives become identical with the continuous ones, thus the continuous approximations becomes exact.

\subsection{Reduced Cosserat model}

In the case when the second derivative of rotations is absent in (5), i.e. when $D_{1}=0$ or $E_{1}-2(h / d)^{2} E_{2}=0$, we have so-called reduced Cosserat model.

The equations of the reduced Cosserat model have the following form

$$
\begin{gathered}
\frac{v_{k+1}-2 v_{k}+v_{k-1}}{h^{2}}-\frac{\varphi_{k+1}-\varphi_{k-1}}{2 h}=0, \\
D_{2}\left(\frac{v_{k+1}-v_{k-1}}{2 h}-\varphi_{k}\right)=0 .
\end{gathered}
$$

Below, we would like to mentions several interesting properties of this model.

In the long-wavelength approximation these equations lead to continuous reduced Cosserat model

$$
v_{x x}-\varphi_{x}=0, \quad v_{x}-\varphi=0,
$$

which, again, can be derived from eq. (6) of the full model by putting $D_{1}=0$.

The general solution has the form

$$
\varphi(x)=v_{x},
$$

$v(x)$ is an arbitrary double differential function.

Thus, the reduced model exhibits some arbitrariness, compared with the solution (7) of the full model. By choosing $v(x)$ being a polynomial

$$
v(x)=C_{1}+C_{2} x+C_{3} x^{2}+C_{4} x^{3},
$$

from eq. (13) we can obtain

$$
\varphi(x)=C_{2}+2 C_{3} x+3 C_{4} x^{2} .
$$

The integration constants can be found from the boundary conditions

$$
v(0)=0, \quad 2 b^{2} D_{2}(v(h) / h-\varphi(0))+M=0, \quad v(L)=0, \quad \varphi(L)=0 .
$$

After finding the integration constants, the final solution can be written in the following form

$$
v(x)=\frac{1}{2 b^{2}} \frac{M x(L-x)^{2}}{h(2 L-h) D_{2}}, \quad \varphi(x)=\frac{1}{2 b^{2}} \frac{M(L-x)(L-3 x)}{h(2 L-h) D_{2}},
$$

which coincides with the solution of the full model (11) for $D_{1}=0$.

\section{Numerical results}

To demonstrate the validity of our approach we have performed the direct numerical simulations of some structures and compared the results of different models.

Let's consider a finite $1 \mathrm{D}$ chain of $2 N+1$ particles with fixed particles at the boundaries in viscous medium with symmetric couplings (see Fig. 1) and momentum $M$ applied to the central particle. 
First, we find the dependence of steady state displacements and rotations of the particles on the applied momentum $M$ by using a relaxation method. The applied momentum load gradually increased in step-wise manner. At each step we solved the set of dynamic equations $(1-3)$. Due to presence of viscosity after certain time all oscillations damped out and the steady state was reached by the system.

The simulations were performed for characteristic dimensionless set of parameters $E_{1}=1, E_{2}=E_{1}, h=1$, $a=b=0.2 h$, i.e. the stiffnesses of all links are the same, the particles are of square shape and their size is much smaller compared with the distance between their centers. In our simulations a finite 1D chain consists of seven particles.

The dependence of the angle of rotation $\varphi$ of the central particle on the dimensionless momentum $\bar{M}$ for different models is shown in Fig. 2, where circles represent the discrete values of the load for which the simulations were performed. Lines between them are shown for eye-tracing only. Note, that the results for all three models agree quite well for small applied moments and induced angles. The sine-Cosserat model (2) has the same accuracy as the standard linear Cosserat model (3). But, the error of the model (2) increases compared with the error of the model (3). However, it is important to note, that the linear Cosserat model (3) does not support continuous rotations at all, while sine-Cosserat model (2) does.

Stars in Fig. 2 indicate the conditions when the central particle starts to rotate. The dynamics of the system calculated via sine-Cosserat (2) and nonlinear (1) models is shown in Fig. 3a and Fig. 3b, respectively. For better visibility, the centers of the particles are connected to show the snapshot of the chain at a given moment of time. The central particle is not shifted. Note, that since the model (2) is linear with respect to displacements, the equations for the longitudinal displacements are not coupled to the transverse one and rotations. Thus, the particles can exhibit the transverse oscillations only (see Fig. 3a). In the nonlinear model (3) all degrees of freedom are coupled to each other, which implies that small oscillations can now induce both transverse as well as longitudinal displacements (see Fig. 3b).

To confirm the localization effect, a test simulation was performed when the central particle exhibited 1500 full body rotations, while other particles performed small oscillations only.

\section{Conclusions}

In the current paper we presented and discussed several models with different degrees of approximation. It was shown, that one of them (1) supports both small oscillational and continuous rotations excitations, but is very cumbersome to use to derive the continuous model. It might still be possible, though, by using operator approach. Another model (3) is a discrete analogue of the equations of motion and the potential energy of the classical micropolar theory [4]. As it was mentioned above, such model was successfully employed to describe systems with small oscillations. But, it doesn't support full continuous rotations. Our aim is to derive models of the generalized continuum mechanics,

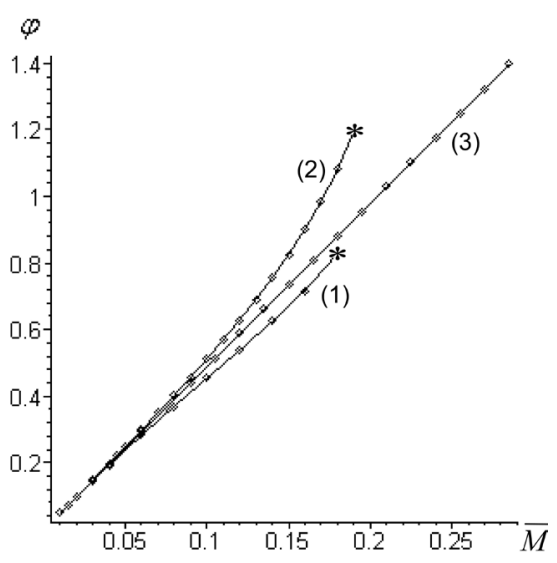

Fig. 2. Dependence of the angle of rotation of the central particle on the applied load for nonlinear (1), sine-Cosserat (2) and standard Cosserat (3) models.

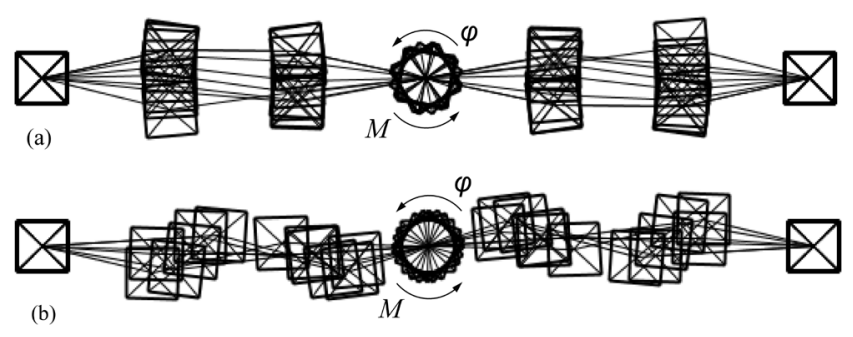

Fig. 3. Demonstration of the dynamics of the system of seven particles with the rotating central particle: sine-Cosserat model (a) and nonlinear model (b).

which can adequately describe continuous rotations as well. In this paper we introduced one more model (2) with sinedependent terms for angles of rotations. The models (2), (3) are linear with respect to displacements. The nonlinearity of the displacements can be taken into account similar to Refs. $[8-11]$.

In Section 3 we derived approximate static solutions for the linear case. In general case, they can be obtained numerically. Since we are interested in the generalized continuum theory, we obtained such solutions based on long-wavelength approximation. Both, full and reduced Cosserat models were discussed. It is important to note, that linear analysis is rather standard approach to study localization effects, which decay with the distance. It is usually used in the study of dynamical effects in lattices $[10,11,12]$, frequency filters $[12,13]$, the tails of localized excitations, such as discrete breathers [14, 15 ] and roto-breathers [16], for the analysis of their existence and properties.

The results of the direct numerical simulations are presented in Section 4. It was shown the good agreement between all discussed models under small loads. Importantly, it was demonstrated, that sine-Cosserat model (2) describes the small static turns of the particles analogous to linear Cosserat model (3), but in addition to this also supports continuous rotation of the particles.

Aknowledgements. The work of AAV was carried out under the financial support of the Ministry of Education and Science of Russian Federation (Project 9.7446.2017/8.9). 


\section{References}

1. A.S. J. Suiker, A. V. Metrikine, R.de Borst. Int. J. Solids Struct. 38, 1563 (2001). DOI: 10.1016/S00207683(00)00104-9

2. K.S. Kim, R. L. Piziali. Int. J. Solids Struct. 23 (11), 1563 (1987). DOI: 10.1016/0020-7683(87)90070-9

3. E. Cosserat, F. Cosserat. Theorie des corps deformables. Hermann, Paris (1909).

4. A.C. Eringen. Theory of micropolar elasticity, in: Fracture, v. 2. Ed. Liebowitz H., New York: Academic Press, 621 (1968).

5. A. A. Vasiliev, S. V. Dmitriev. Russian Physics Journal 59, 961 (2016). DOI: 10.1007/s11182-016-0861-1

6. E. F. Grekova, M. A. Kulesh, G. C. Herman. Bull. Seismol. Soc. Am. 99 (2B), 1423 (2009). DOI: 10.1785/0120080154

7. E. F. Grekova. Doklady Physics 60 (5), 232 (2015). DOI: 10.1134/S1028335815050080

8. I. S. Pavlov, A. I. Potapov, G. A. Maugin. Int. J. Solids Struct. 43, 6194 (2006). DOI: 10.1016/j.ijsolstr.2005.06.012
9. S. A. Lisina, A. I. Potapov, V. F. Nesterenko. Acoust. Phys. 47 (5), 598 (2001). DOI: 10.1134/1.1403551

10. A. V. Porubov, I. E. Berinskii. Int. J. Non-Linear Mech. 67, 27 (2014). DOI: 10.1016/j.ijnonlinmec.2014.07.003

11. A.V. Porubov, I.E. Berinskii. Mathematics and Mechanics of Solids. 21 (1), 94 (2016). DOI: $10.1177 / 1081286515577040$

12. R. K. Narisetti, M. Ruzzene, M. J. Leamy. Journal of Vibration and Acoustics 133 (6), 061020 - 1 (2011). DOI: 10.1115/1.4004661

13. S. Sugino, Y. Xia, S. Leadenham, M. Ruzzene, A. Erturk. Journal of Sound and Vibrations 406, 104 (2017). DOI: 10.1016/j.jsv.2017.06.004

14. S. Flach, A. V. Gorbach. Physics Reports 467(1), 1 (2008). DOI: 10.1016/j.physrep.2008.05.002

15. S.V. Dmitriev, E.A. Korznikova, Yu.A. Baimova, M. G. Velarde. Physics-Uspekhi 59 (5), 446 (2016). DOI: 10.3367/UFNe.2016.02.037729

16. S. Flach, M. Spicci. J. Phys.: Condens. Matter 11, 321 (1999). DOI: 10.1088/0953-8984/11/1/027 